\title{
Influence of menopause on mandibular bone quantity and quality in Japanese women receiving dental implants
}

\author{
M. Munakata $\cdot$ N. Tachikawa $\cdot$ E. Honda $\cdot$ M. Shiota $\cdot$ \\ S. Kasugai
}

Received: 5 January 2011 / Accepted: 3 May 2011 / Published online: 10 June 2011

(C) The Author(s) 2011. This article is published with open access at Springerlink.com

\begin{abstract}
Summary The purpose of this study was to evaluate the effect of menopause on bone mineral density and bone width of the mandible. Results indicate that menopause affects the bone quality and quantity of the partially edentulous molar region of the mandible, which should be considered in dental implant treatment for postmenopausal women.

Introduction The recovery of oral function with dental implant is clinically effective and highly predictable. Bone quantity and quality at the implant installation site affect its prognosis; however, the effects of menopause on jaw bone have not been well documented. The purpose of this study was to evaluate the effect of menopause on bone mineral density (BMD) and bone width of the mandible.

Methods The subjects were 72 female patients with a partially edentulous molar region of the mandible: 30 premenopausal and 42 postmenopausal women aged 30 to 70 years. Trabecular BMD was measured with quantitative computed tomography. Trabecular region width (TW) and cortical width $(\mathrm{CW})$ were measured with $\mathrm{CT}$. The BMD, $\mathrm{TW}$, and $\mathrm{CW}$ of the two groups were compared.

Results The trabecular BMD of postmenopausal women was lower than that of the premenopausal women. The TW
\end{abstract}

M. Munakata $(\bowtie) \cdot$ N. Tachikawa $\cdot$ M. Shiota $\cdot$ S. Kasugai Oral Implantology and Regenerative Dental Medicine,

Department of Masticatory Function Rehabilitation,

Tokyo Medical and Dental University,

1-5-45 Yushima, Bunkyo-ku,

Tokyo 113-8549, Japan

e-mail: munakata.impl@tmd.ac.jp

E. Honda

Oral and Maxillofacial Radiology, Department of Oral and Maxillofacial Dentistry, Tokushima University,

2-24, Shinkura-cho,

Tokushima 770-8501, Japan of postmenopausal women was greater than that of premenopausal women, whereas the $\mathrm{CW}$ of postmenopausal women was significantly smaller than that of premenopausal women. In all these women, BMD correlated negatively with TW and positively with $\mathrm{CW}$. In the premenopausal women, BMD negatively correlated with $\mathrm{TW}$, but it did not correlate with $\mathrm{CW}$. In the postmenopausal women, there was no correlation between BMD and bone width.

Conclusion These results indicate that menopause affects the bone quality and quantity of the partially edentulous molar region of the mandible, which should be considered in dental implant treatment for postmenopausal women.

Keywords Bone mineral density · Bone quality · Bone quantity · Dental implant · Menopause · Quantitative computed tomography

\section{Introduction}

Loss of posterior teeth may result in the loss of neuromuscular stability of the mandible, reduced masticatory efficiency, loss of vertical dimension of occlusion, and poor aesthetics. Prosthetic rehabilitation should aim at restoring the vertical dimension and increasing the occlusal contact area in the premolar/molar region. Dental implant treatment is an excellent option for prosthetic restoration that is associated with high success rates. Bone quantity and quality are very important for successful implant treatment. Of these two, a vital determinant for implant placement feasibility and the potential for bone grafting is bone quantity, which can now be determined more precisely by the various X-ray CT systems including helical $\mathrm{CT}$ and small radiation field $\mathrm{CT}$ and the wide 
variety of software available for image analyses and multiplanar reconstruction.

Bone quality and cortical bone width, which are also important factors affecting primary implant stability and the duration of the non-loading period, have been classified by Lekholm et al. [1] and Misch [2], and their classification systems have been widely used in clinical practice. However, the practical and substantial assessment of bone quality often depends on the tactile impression while drilling to prepare the implant site and visual evaluation of X-ray CT and topographic images, which should be regarded as subjective procedures. Existing methods able to objectively assess bone quality depend on the cutting resistance experienced while preparing the implant site and the insertion torque during implant insertion. These are unable to provide a good preoperative understanding of bone quality and are sensitive to the procedures of implant surgery as well as implant geometry [3]. CT values obtained by X-ray scanning are another evaluation index of bone quality which, however, are not reliable in quantitative terms completely quantitative as $\mathrm{CT}$ values are influenced by the type of CT system used, tube voltages, algorithms, etc. In addition to the methods described above, a convenient and helpful method using panoramic radiography is available to assess the jaw bone, but it does not provide quantitative data.

We have applied a quantitative computed tomography (QCT) method, which was developed by Genant et al. [4] and has been widely used in the lumbar vertebrae and femur for the diagnosis of osteoporosis, to the quantitative and objective assessment of jaw bone quality and reported the changes in mandibular bone quality.

The purpose of this study was to investigate the effect of menopause on mandibular bone quantity and quality in the edentulous molar region in patients receiving dental implant.

\section{Subjects and methods}

The present study was approved by the Dental Hospital at Tokyo Medical and Dental University Ethics Committee and the written informed consents were obtained from all the patients.

\section{Subjects}

The subjects were 72 Japanese women with a partially edentulous molar region of the mandible receiving dental implant at the Dental Hospital at Tokyo Medical and Dental University between 2005 and 2009. Thirty of these subjects were premenopausal and 42 were postmenopausal. They were aged from 40 to 78 years. Exclusion criteria were: impaired bone metabolism (such as hyperparathyroidism, renal osteodystrophy, and oophorectomy, skeletal bone disorders) and the use of medications that affect bone metabolism (such as corticosteroids, estrogen preparation, bisphosphonate, and vitamin D).

Bone mineral density and bone width measurement

The trabecular bone mineral density (BMD) of the mandible was measured using single-energy QCT with a Siemens Somatom plus S (Siemens, Germany) at $120 \mathrm{kV}$ and $125 \mathrm{~mA}$ with a slice thickness of $1 \mathrm{~mm}$.

1. The mandibular inferior border was placed vertical to the table with the mouth closed and set as the reference plane to perform $\mathrm{CT}$ scanning.

2. The simultaneous scanning of a bone density calibration phantom containing two compartments with 0 and $200 \mathrm{mg} / \mathrm{ml}$ of calcium hydroxyapatite equivalent was performed when imaging in patients was done (Fig. 1).

3. Among all the CT images obtained, the sliced image containing the mental foramen parallel to the mandibular inferior border was selected for the measurement (Fig. 2).

4. The edentulous region in the abovementioned image was set as the region of interest (ROI) to determine the average CT values of the trabecular bone in the edentulous molar region. The regions where the CT

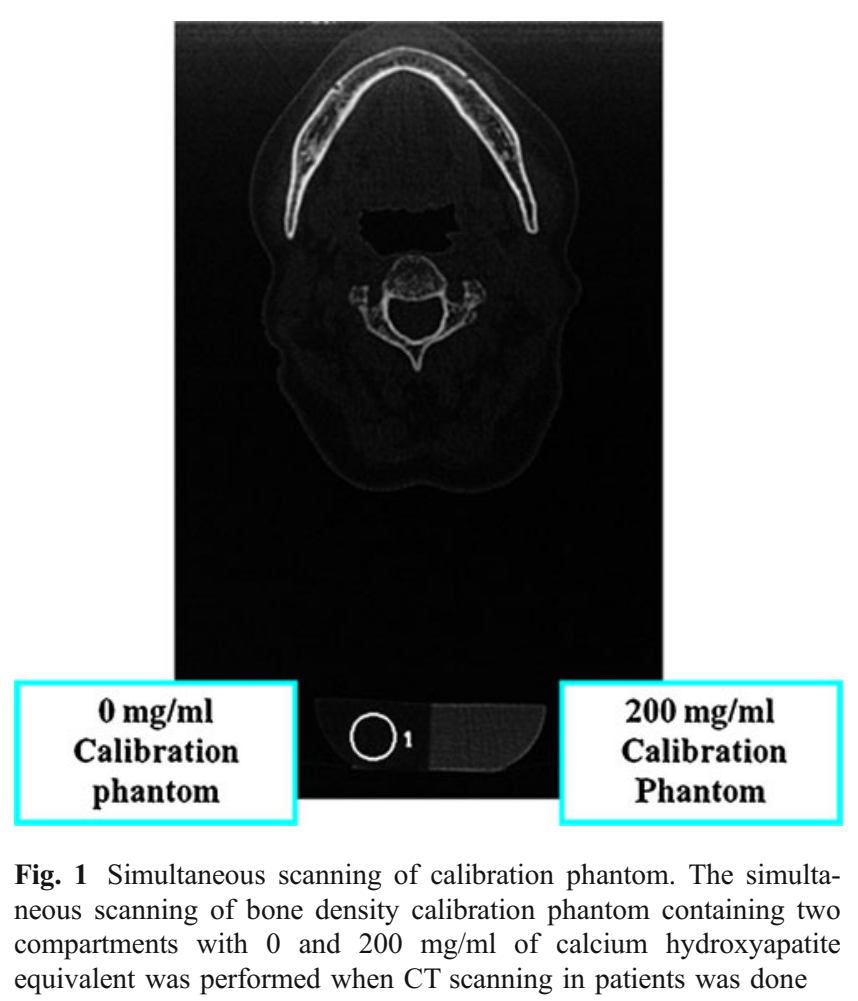




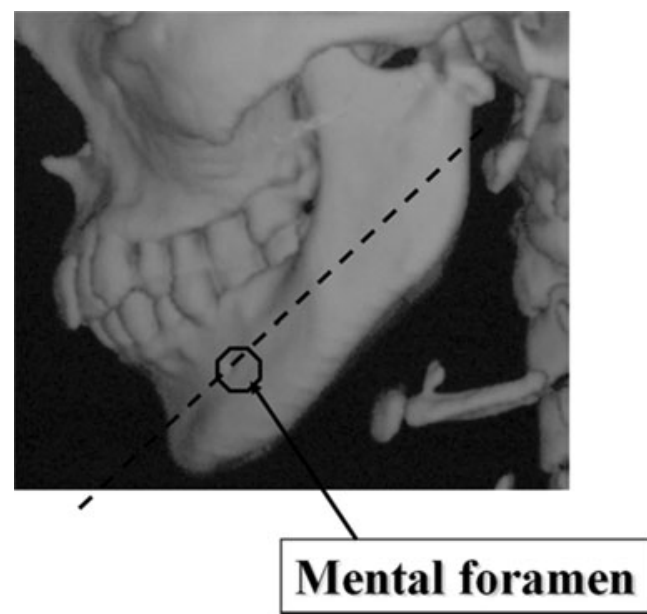

Fig. 2 Image selection for measurements. The sliced images containing the mental foramen parallel to the lower border of the mandible were selected

values were $<500 \mathrm{HU}$ were regarded as trabecular bone regions.

5. Those parts presenting with localized bone sclerosis within the image of the trabecular bone region were excluded from CT value measurement. The areas adjacent to the interface between cortical and trabecular bone were also excluded from the ROI to eliminate the partial volume effect. The $\mathrm{CT}$ values measured within the ROI $(3 \times 3 \mathrm{~mm})$ three times were averaged to obtain mean $\mathrm{CT}$ values for individual trabecular bone regions. These mean $\mathrm{CT}$ values and the $\mathrm{CT}$ values obtained with the calibration phantom were converted based on the linear relationship between the X-ray attenuation coefficients and the CT values to calculate the values of bone mineral density (in milligrams per milliliter: BMD).

6. Additionally, for the edentulous molar region in the same sliced image (bone mode), the widths of the trabecular bone region (TW) as well as the width of the cortical bone $(\mathrm{CW})$ including those in both the buccal and lingual layers of the cortex sides were determined using the distance measurement software provided with the CT scanner (Fig. 3).

\section{Statistical analysis}

The following three analyses were performed using the measurements of the bone widths and the calculated BMD values:

1. The comparison of the BMD values and the bone widths between pre- and postmenopause

2. The correlations between BMD and bone width

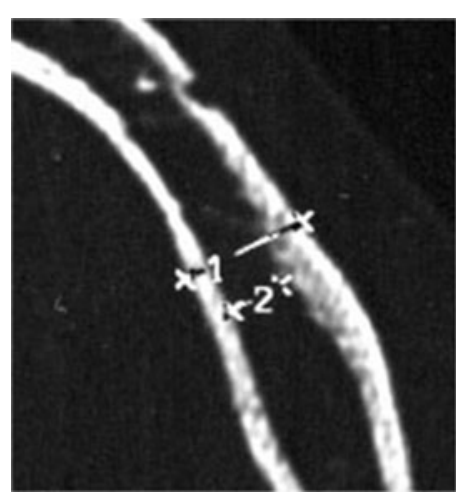

1-2: Cortical bone width

2: Trabecular bone width

The Wilcoxon test was used to verify possible differences between two groups and the correlations were determined using Spearman's rank correlation coefficient. Statistical significance was accepted at the $P<0.005$ level.

\section{Results}

Comparison of the BMD values and bone widths

between pre- and postmenopausal women

Table 1 shows the values of BMD and bone widths of premenopausal and postmenopausal women.

The BMD values of postmenopausal women were significantly lower. Significantly higher TW values and lower CW values were observed in postmenopausal women. Thus, the results suggest a reduced percentage of cortical bone in the mandible of postmenopausal women.

Correlations between BMD and bone width

Figures 4, 5, and 6 shows the correlation between BMD and bone width (TW and CW) for all women (Fig. 4), premenopausal women (Fig. 5), and postmenopausal women (Fig. 6), respectively.

For all women $(n=72)$, BMD correlated negatively with TW $(r=-0.560)$ and positively with $\mathrm{CW}(r=0.593)$. For

Table 1 Comparison of bone mineral density and bone width between pre- and postmenopausal women

\begin{tabular}{llll}
\hline & BMD (mg/ml) & TW (mm) & CW (mm) \\
\hline Premenopausal women & $222 \pm 88^{* *}$ & $6.5 \pm 1.7^{* *}$ & $5.5 \pm 1.8^{* *}$ \\
Postmenopausal women & $109 \pm 69$ & $8.7 \pm 1.6$ & $3.9 \pm 1.1$
\end{tabular}

$* * p<0.005$ 


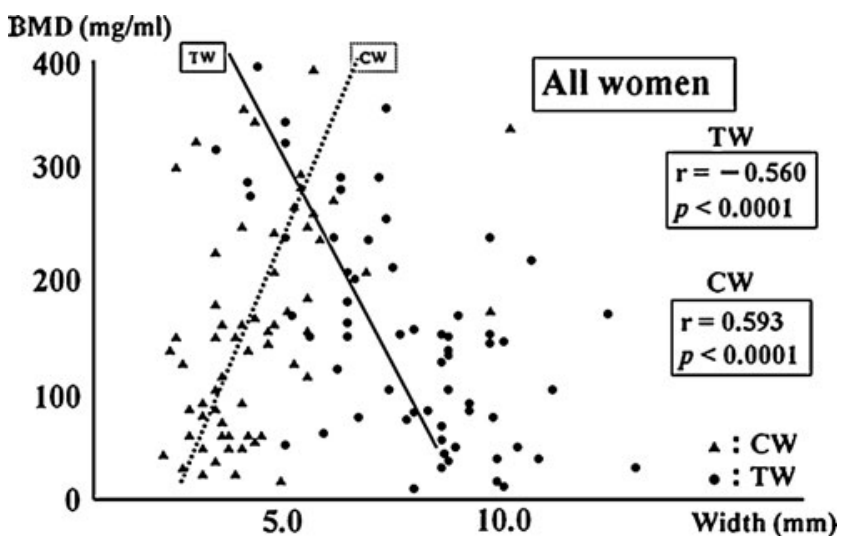

Fig. 4 Correlation between bone width and bone mineral density for all patients. $T W$ and $C W$ represent bone width of trabecular bone and cortical bone, respectively

premenopausal women, BMD correlated negatively with TW $(r=-0.592)$ but has no correlation with CW. No correlations between BMD and bone width were observed for postmenopausal women.

\section{Discussion}

Utility of QCT technique in preoperative assessment for implant surgery

Conventional quantitative assessment methods for the jaw bone include the panoramic-based measurement method introduced by Benson et al. [5], photodensitometry using intraoral radiography, and dual energy X-ray absorptiometry (DXA) [6, 7]. These modalities have been used in several previous studies. QCT used in this study determines bone quantity using a three-dimensional measurement method by means of X-ray CT. This has several advantages over other

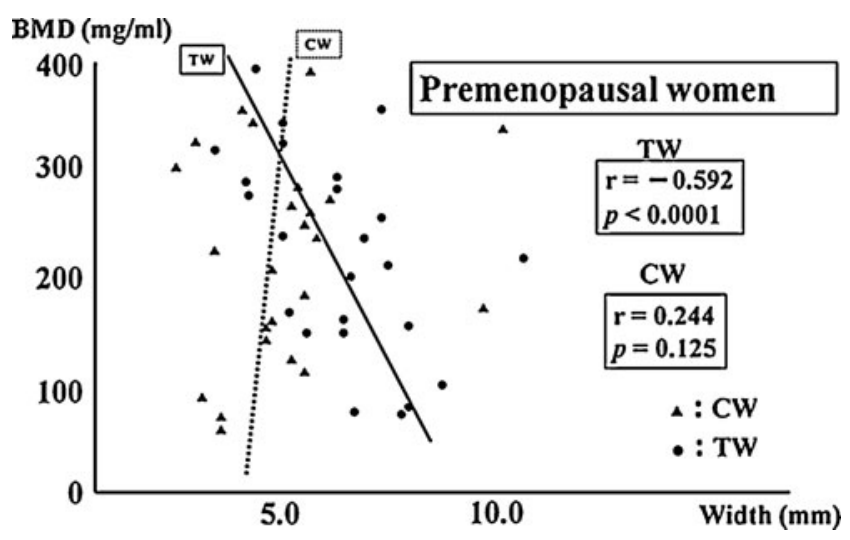

Fig. 5 Correlation between bone width and bone mineral density for premenopausal women

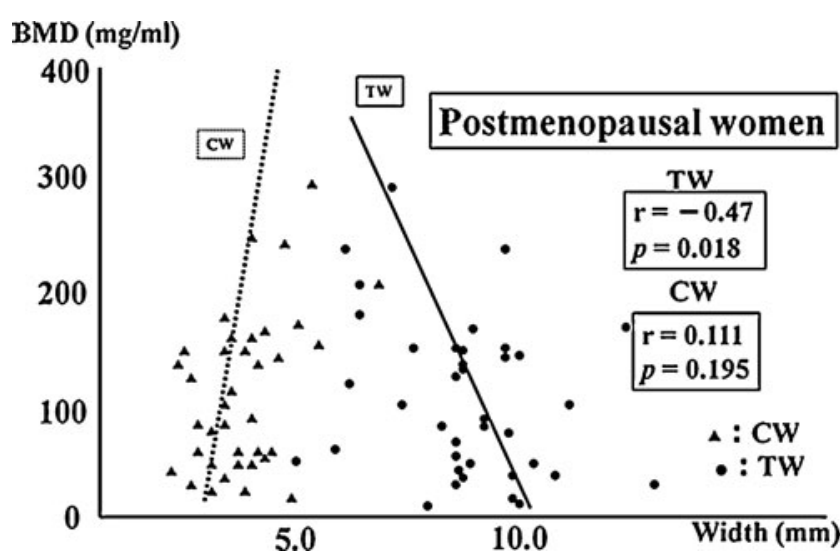

Fig. 6 Correlation between bone width and bone mineral density for postmenopausal women

measurement methods including the individual measurement of trabecular and cortical bone and the possible determination of bone density per volume (in milligrams per milliliter), and thus it may be the measurement method of choice for the jaw bone, given its complex and uneven structure. However, the QCT method has the disadvantages of relatively high exposure dose compared to that required for other radiography methods and its poor reproducibility due to high sensitivity to patient positioning and ROI setting. However, these disadvantages can be virtually eliminated as only a single exposure is required when applying the QCT method to preoperative assessment for implant surgery. By a single scan with the additional operation of simply mounting the ready-made calibration phantom onto the head, the QCT method can provide quantitative bone quality measurements of both cortical and trabecular as well as information including bone morphology and quantity at the planned implant site. Thus, it is an extremely helpful modality for preoperative examination prior to implant.

Furthermore, as the jaw bone can be affected by retained teeth and local impacts including mechanical stimulation caused by occlusion, careful consideration should be given to the measurement site within the jaw to be set. Therefore, patients with a posterior free end missing were included in this study to minimize the possible effects of such factors.

Influences of menopause on mandibular bone

In the studies of the influences of menopause on all the bones of the body by Ito et al. [8], Kroger et al. [9], and Kin et al. [10], the evident loss of bone mass and BMD after menopause have been reported for lumbar vertebral bodies and the femur.

Irie et al. [11] investigated the effect of menopause on mandibular bone by examining three-dimensional morpho- 
logical changes in the mandibular bone of ovariectomized rats using micro $\mathrm{CT}$ and reported that not only significantly decreased values for both bone mass and width of trabecular bone but also significantly increased distances between trabeculae were observed in ovariectomized rats compared to controls. Kuroda et al. [12] also performed a similar study using DXA and pQCT methods and reported a significantly decreased trabecular bone density in the posterior region of the mandible in the ovariectomized rats compared to controls while no difference was observed for the cortical bone density in the same region. Furthermore, Kurosu et al. [13] investigated the correlation between the bone density of lumbar vertebrae determined by DXA examination and that of mandibular bone measured using QCT in 30 postmenopausal women with teeth and reported that a positive correlation existed between the bone density of lumbar vertebrae and the trabecular bone density of mandibular bone. Meanwhile, Klemetti et al. [14] conducted a similar study in 71 postmenopausal women with edentulous or partially edentulous jaws using QCT and reported that there was no correlation between the bone density of the lumbar vertebrae and density of trabecular bone in the mandible, but a correlation with mandibular cortical bone density existed. In addition, the results of the study by Taguchi et al. [15] using QCT were consistent with Klemetti's findings.

In this study, the BMD values for mandibular trabecular bone in the edentulous region were significantly lower in postmenopausal women than in premenopausal women. The disagreement between our results and those of Klemetti et al. [14] and Taguchi et al. [15] may be attributed to the different patient histories from those in the studies of them as half of the patients that are included in our study had only a small edentulous span with three or fewer missing teeth. Thus, the possible contribution of occlusal forces derived from adjacent teeth to such results was suggested. However, in this study, the influences of other factors including the number of residual teeth, age, and the number of years after menopause were not taken into consideration and should be adequately investigated in the future.
Moreover, our study revealed that a significantly large proportion of the edentulous region of the mandible consisted of trabecular bone while the proportion occupied by cortical bone was significantly small. Taguchi et al. [16] reported a significant correlation between the bone density of lumbar vertebrae and cortical thickness and the morphology of the mandibular lower border, a finding which supports the results from our study together with the abovedescribed results of Irie et al. [11].

Therefore, in the mandible where teeth are lost, the bone density may be reduced and the loss of trabecular bone density as well as cortical bone resorption from the inside can advance with menopause just as they do in bones in other parts of the body.

\section{Relationships between bone quality and quantity}

Osteoporosis is a systemic skeletal disease characterized by low bone mass and micro-architectural deterioration of bone tissue leading to enhanced bone fragility and an increase in fracture risk [17]. This bone fragility involves both bone quantity and quality; specifically, it results from thinning of the cortical bone and cavity expansion due to bone absorption at the endosteum surface as well as reduced bone density.

Regarding osteoporosis of the mandible, Tamai et al. [18] investigated mandibular bone quality using QCT in healthy subjects and reported that the BMDs of mandibular cortical and cancellous bone reached a peak when subjects were in their $30 \mathrm{~s}$ and $40 \mathrm{~s}$ and then began to decline. Takaishi et al. [19] also reported that mandibular alveolar bone mineral density diminishes with age. With regard to bone quantity, Von Wowern et al. [20] reported that in the mandible, the cortical bone mass diminishes with age. On the other hand, Kribbs et al. [21] showed that diminution of the mandibular mass is not correlated with age.

From our study results, the BMD of trabecular bone correlated negatively with trabecular bone width and positively with cortical bone width and such tendency was more distinctly observed in women. Therefore, these

Table 2 Classification of mandibular bone for implant treatment

\begin{tabular}{|c|c|c|c|c|c|}
\hline Class & A & $\mathrm{B}$ & $\mathrm{C}$ & $\mathrm{D}$ & $\mathrm{E}$ \\
\hline Premenoposal women (30cases) & 7 & 21 & 0 & 2 & 0 \\
\hline Postmenoposal women (42cases) & 0 & 10 & 15 & 12 & 5 \\
\hline Thickness of cortical bone (mm) & $>3.5$ & $3.5>,>1$ & $3.5>,>1$ & $1>$ & $1>$ \\
\hline $\mathrm{BMD}(\mathrm{mg} / \mathrm{ml})$ & $>300$ & $300>,>80$ & $80>$ & $300>,>80$ & $80>$ \\
\hline
\end{tabular}

Class $A$ thick cortical bone and limited medullary cavity (or much trabecular bone); class $B$ thick cortical bone but medullary cavity (or less trabecular bone); class $C$ thin cortical bone but limited medullary cavity (or much trabecular bone); class $D$ thin cortical bone and medullary cavity (or less trabecular bone); class $E$ thin cortical bone and medullary cavity (or less trabecular bone) 
findings indicate that the reduced bone strength in the mandible develops with age just as in the other bones in the body, caused by age-associated BMD loss of mandibular trabecular bone accompanying increased trabecular bone mass and reduced cortical bone mass. It would also appear that trabecular bone quality reflects bone quantity in the same area.

New classifications of jaw bone for implant treatment

For predictable implant treatment, a number of patientrelated factors need to be understood. These include systemic conditions such as a history of diabetes, etc., oral hygiene, periodontal condition, smoking habit, jaw bone quality and quantity, occlusion patterns, and parafunctional habits. As bone quality is a crucial factor for the successful achievement of osseointegration, systems for its classification have been developed by Lekholm et al. [1] and Misch [2], and based on their classification, many studies and case reports have been published to date. However, classification based on visual assessment of radiographic images is associated with several problems including imaging stability, which is affected by the tube voltage and current of the X-ray unit and degradation of the developing fluid. Moreover, assessment based on the tactile sensation during implant site preparation has been questioned due to poor objectivity as reported in the study by Trisi et al. [22].

Clinically, cortical bone thickness significantly affects implant primary stability compared to the trabecular bone. Thus, we measured the thickness of cortical bone and BMD and classified jaw bone for implant treatment (Table 2). Two patients of all participants, who experienced loose implants or early bone resorption during the nonloading period after implant insertion, were both premenopausal women whose bone quality was classified into A with high BMD values. Therefore, these failures may be attributable to heat-induced injury during implant site preparation and placement. Thus, we can conclude that these results have demonstrated the usefulness of the quantitative measurement of bone density for implant treatment and the utility of new bone classification based on such measurements.

Our classification using QCT may have applications beyond implant treatment planning based on the preoperative objective assessment of bone quality. It may also be possible to use it for inter-patient comparison and the evaluation of grafted bone. The correlation between our classification and factors including cutting resistance and removal torque should be further investigated to improve its clinical utility.

\section{Conflicts of interest None.}

Open Access This article is distributed under the terms of the Creative Commons Attribution Noncommercial License which permits any noncommercial use, distribution, and reproduction in any medium, provided the original author(s) and source are credited.

\section{References}

1. Lekholm U, Zarb GA (1985) Patient selection and preparation. In: Branemark PI, Zarb GA, Albrektsson T (eds) Tissue-integrated prosthesis: osseointegration in clinical dentistry. Quintessence, Chicago, pp 199-209

2. Misch CE (1993) Density of bone: effect on treatment planning, surgical approach, and healing. In: Contemporary implant dentistry. Mosby, St. Louis, pp 469-485

3. Johanson P, Strid KG (1994) Assessment of bone quality from cutting resistance during implant surgery. Int J Oral Maxillofac Implants 9:279-288

4. Genant HK, Cann CE, Ettinger B, Gorden GS (1982) Quantitative CT of vertebral spongisa: a sensitive method for detecting early bone loss after oophorectomy. Ann Intern Med 97:699-705

5. Benson BW, Phihoda TJ, Glass BJ (1991) Variations in adult cortical bone mass as measured by a panoramic mandibular index. Oral Surg Oral Med Oral Pathol 71:349-356

6. Von Wowern N (1988) Bone mineral content of mandibles: normal reference values-rate of age-related bone loss. Calcif Tissue Int 43:193-198

7. Bras J, van Ooij CP, Abraham-Inpijin L, Kusen GJ, Wilmink JM (1982) Radiographic interpretation of the mandibular angular cortex: a diagnostic tool in metabolic bone loss. Part 1. Normal state. Oral Surg Oral Med Oral Pathol 53:541-545

8. Ito M, Hayashi K, Kawahara Y, Uetani M, Imaizumi Y (1993) The relationship trabecular and cortical bone mineral density to spinal fractures. Invest Radiol 28:573-580

9. Kroger H, Tuppurainen M, Honkanen R, Alhava E, Saarikoski S (1994) Bone mineral density and risk factors for osteoporosis: a population-based study of 1,600 perimenopausal women. Calcif Tissue Int 55:1-7

10. Kin K, Kushida K, Yamazaki K, Okamoto S, Inoue T (1991) Bone mineral density of the spine in normal Japanese subjects using dual energy X-ray absorptiometry. Calcif Tissue Int 55:324-329

11. Irie M, Sakakura Y, Tsuruga E, Hosokawa Y, Yajima T (2004) Three-dimensional changes of the mandible and alveolar bone in the ovariectomized rat examined by micro-focus computed tomography. J Jpn Soc Periodontol 46:288-293

12. Kuroda S, Mukohyama H, Kondo H, Aoki K, Ohya K, Ohyama T, Kasugai S (2003) Bone mineral density of the mandible in ovariectomised rats: analyses using dual energy x-ray absorptiometry and peripheral quantitative computed tomography. Oral Dis 9:24-28

13. Kurosu K, Inagaki K, Yoshinari N, Sato R, Fuma D, Sakano M, Sugiishi T, Iwata T, Fukada J, Takagi Y, Noguchi T, Naitoh M, Ariji E, Taguchi A (2004) Relationship between the bone mineral density of mandible by quantitative computed tomography and lumbar spine by dual energy X-ray absorptiometry. J Jpn Soc Periodontol 46:202-208

14. Klemetti E, Vanio P, Lassila V, Alhava E (1993) Cortical bone mineral density in the mandible and osteoporosis status in postmenopausal women. Scand J Dent Res 101:219-223

15. Taguchi A, Tanimoto K, Suei Y, Otani K, Wada T (1996) Relationship between the mandibular and lumbar vertebral bone mineral density at different postmenopausal stages. Dentomaxillofac Radiol 25:130-135

16. Taguchi A, Suei Y, Ohtsuka M, Otani K, Tanimoto K, Ohtaki M (1996) Usefulness of panoramic radiography in the diagnosis of postmenopausal osteoporosis in women: width and morphology of inferior cortex of the mandible. Dentomaxillofac Radiol 25:263-267 
17. NIH Consensus Development Panel on Osteoporosis Prevention, Diagnosis, and Therapy (2001) Osteoporosis prevention, diagnosis, and therapy. JAMA 285:785-795

18. Tamai M, Ishii Y (1996) The study on changes of bone mineral content of mandible by quantitative computed tomography. J Jpn Stomatol Soc 45:97-104

19. Takaishi Y, Okamoto Y, Ikeo T, Morii H, Takeda M, Hide K, Arai T, Nonaka K (2005) Correlations between periodontitis and loss of mandibular bone in relation to systemic bone changes in postmenoposal Japanese women. Osteoporos Int $16: 1875-1882$

20. Von Wowern N, Stoltze K (1980) Pattern of age related bone loss in mandibles. Scand J Dent Res 88:134-146

21. Kribbs PJ, Chesnut CH 3rd, Ott SM, Kilcoyne RF (1990) Relationship between mandibular and skeletal bone in a population of normal women. J Prosthet Dent 63:86-89

22. Trisi P, Rao W (1999) Bone classification: clinical-histomorphometric comparison. Clin Oral Implants Res 10:1-7 